\title{
A Demons Algorithm for Image Registration with Locally Adaptive Regularization
}

\author{
Nathan D. Cahill ${ }^{1,2}$, J. Alison Noble ${ }^{1}$, and David J. Hawkes ${ }^{3}$ \\ 1 Institute of Biomedical Engineering, University of Oxford, Oxford, UK \\ 2 Research and Innovation, Carestream Health, Inc., Rochester, NY, USA \\ 3 Centre for Medical Image Computing, University College London, London, UK
}

\begin{abstract}
Thirion's Demons [1] is a popular algorithm for nonrigid image registration because of its linear computational complexity and ease of implementation. It approximately solves the diffusion registration problem [2] by successively estimating force vectors that drive the deformation toward alignment and smoothing the force vectors by Gaussian convolution. In this article, we show how the Demons algorithm can be generalized to allow image-driven locally adaptive regularization 314] in a manner that preserves both the linear complexity and ease of implementation of the original Demons algorithm. We show that the proposed algorithm exhibits lower target registration error and requires less computational effort than the original Demons algorithm on the registration of serial chest CT scans of patients with lung nodules.
\end{abstract}

\section{Introduction}

At a high level, image registration algorithms can be described by three components: a cost function that describes the dissimilarity between two images, a space of geometric transformations under which one or both images are allowed to deform, and, a strategy for minimizing the cost function over the space of allowable transformations. In nonparametric registration [2, the set of vectorvalued functions $\left\{\boldsymbol{\Phi}: \mathbb{R}^{n} \mapsto \mathbb{R}^{n}\right\}$ forms the allowable space of transformations. In order to guarantee that the nonparametric registration problem is well-posed and has a smooth solution, a regularization term must be added to the dissimilarity measure to form the cost function.

In medical image registration, various regularizers, such as elastic, fluid, diffusion, and curvature have been proposed [2] for inclusion into the cost function, each defining a notion of smoothness in a slightly different way. A variety of different techniques [1 2566] have been proposed for numerically solving the Euler-Lagrange equations arising from the variational minimization of these cost functions. In this article, we focus on Thirion's Demons algorithm [1, which is a popular choice because of its linear complexity and simple implementation.

Thirion's Demons algorithm approximates the solution to nonparametric registration with a homogeneous diffusion regularizer by iteratively performing two steps: 1) computing a force vector that corresponds to the variational derivative of the dissimilarity measure, and 2) convolving the force vector with a Gaussian 
kernel. The Demons algorithm can be generalized for use with curvature and fluid regularizers as described in [8, by reformulating the solution of the EulerLagrange equation as the stationary solution of a coupled system of diffusion equations.

Each of the above-mentioned regularizers are homogeneous and isotropic, meaning they ensure smoothness independently of location or direction. In the optic flow community, much research has been done into generalizing diffusion regularization to be nonhomogeneous and anisotropic [4. Even though adaptive diffusion regularizers allow for greater flexibility, the resulting Euler-Lagrange equations are not of a form that fits nicely into the framework of an efficient, easy to implement Demons-style algorithm.

In this paper, we show that a Demons-style algorithm that uses successive Gaussian convolution can be constructed to handle image-driven locally adaptive regularization. This is possible if the locally adaptive regularizer is based on a generalization of the curvature regularizer instead of the diffusion regularizer. When the proposed locally adaptive curvature regularizer is incorporated in a variational registration problem, the resulting Euler-Lagrange equation can be linked to a coupled system of diffusion equations whose stationary solution can be approached by Demons-style successive Gaussian convolution. We illustrate the behavior of the proposed algorithm on a pedagogical example and on serial CT chest exams.

\section{Background}

Consider two images, a reference image $R$ and a floating image $F$, both as functions in $\mathbb{R}^{n}$. Define a deformation $\boldsymbol{\Phi}: \Omega \subset \mathbb{R}^{n} \mapsto \mathbb{R}^{n}$ by $\mathbf{\Phi}(\mathbf{x})=\mathbf{x}-\mathbf{u}(\mathbf{x})$, and call $\mathbf{u}$ the displacement. The general form of the registration problem is given by:

$$
\min _{\mathbf{u}} \mathcal{E}(R, F, \mathbf{u}):=\mathcal{S}(\mathbf{u})+\alpha \mathcal{J}\left(R, F^{\mathbf{u}}\right),
$$

where $\mathcal{J}$ is a dissimilarity measure that quantifies the dissimilarity between the reference image $R$ and the deformed floating image $F^{\mathbf{u}}:=F(\boldsymbol{\Phi}), \mathcal{S}$ is a regularizer that ensures that the minimization problem is well-posed and that the solution is smooth in some sense, and $\alpha$ is a weighting parameter.

Necessary conditions for a minimum of $\mathcal{E}(R, F, \mathbf{u})$ are given by the EulerLagrange equations:

$$
\mathcal{A}(\mathbf{u}(\mathbf{x}))=\alpha \mathbf{f}(\mathbf{x}, R, F, \mathbf{u}(\mathbf{x})),
$$

with suitable boundary conditions. The partial differential operator $\mathcal{A}$ and force vector $\mathbf{f}$ arise from the Gâteaux derivatives of the regularizer and dissimilarity measure, respectively. In this article, we use the negative of the squared correlation coefficient $(\mathrm{CC})$ dissimilarity measure, which yields the force vector:

$$
\mathbf{f}(\mathbf{x}, R, F, \mathbf{u}(\mathbf{x}))=-\frac{2 \rho_{R, F \mathbf{u}}^{2}}{|\Omega|}\left[\left(R(\mathbf{x})-\mu_{R}\right)-\frac{\left(F^{\mathbf{u}}(\mathbf{x})-\mu_{F \mathbf{u}}\right)}{\sigma_{F \mathbf{u}}^{2}}\right] \nabla F^{\mathbf{u}}(\mathbf{x}),
$$


where $\mu_{A}, \sigma_{A}^{2}$, and $\rho_{A, B}$ refer to the mean, variance, and correlation coefficient, respectively. We note that our subsequent analysis is not dependent on this choice of $\mathcal{J}$.

The diffusion and curvature regularizers 2 and their corresponding partial differential operators given by:

$$
\begin{aligned}
\mathcal{S}_{\text {diff }}(\mathbf{u})=\frac{1}{2} \int_{\Omega} \sum_{j=1}^{n}\left\|\nabla u_{j}(\mathbf{x})\right\|^{2} d \mathbf{x}, & \mathcal{A}_{\text {diff }}(\mathbf{u}(\mathbf{x}))=-\Delta \mathbf{u}(\mathbf{x}), \\
\mathcal{S}_{\text {curv }}(\mathbf{u})=\frac{1}{2} \int_{\Omega} \sum_{j=1}^{n}\left(\Delta u_{j}(\mathbf{x})\right)^{2} d \mathbf{x}, & \mathcal{A}_{\text {curv }}(\mathbf{u}(\mathbf{x}))=\Delta^{2} \mathbf{u}(\mathbf{x}) .
\end{aligned}
$$

For large deformation problems, these partial differential operators can be applied to the velocity field $\mathbf{v}(\mathbf{x}, t)$ of the deformation, which is related to the displacement field by the material derivative:

$$
\mathbf{v}(\mathbf{x}, t)=\partial_{t} \mathbf{u}(\mathbf{x}, t)+(\nabla \mathbf{u}(\mathbf{x}, t))^{\mathrm{T}} \mathbf{v}(\mathbf{x}, t) .
$$

\subsection{Locally Adaptive Diffusion Registration}

Image-driven adaptive diffusion regularization can be enabled by applying a scalar weighting function to the diffusion regularizer; i.e.

$$
\mathcal{S}_{\text {diff }}^{\text {idadapt }}(\mathbf{u})=\frac{1}{2} \int_{\Omega} \beta^{\mathbf{u}}(\mathbf{x})\left(\sum_{j=1}^{n}\left\|\nabla u_{j}\right\|^{2}\right) d \mathbf{x} .
$$

The weighting function $\beta(\mathbf{x})$ can be chosen based on the type of behavior desired. Alvarez [3] proposes using a weighting function that is inversely proportional to the gradient magnitude of the underlying image. Charbonnier [9] and Bruhn [10] show that a function fitting this description is $\beta(\mathbf{x})=\Psi_{\epsilon}\left(\|\nabla F(\mathbf{x})\|^{2}\right)$, where

$$
\Psi_{\epsilon}\left(s^{2}\right)=\frac{\epsilon}{\sqrt{s^{2}+\epsilon^{2}}} .
$$

Kabus [11 proposes a different type of weighting function that is based on a segmentation of the image into foreground/background regions. He also allows the weighting function to deform over the course of registration according to $\mathbf{u}(\mathbf{x})$. Here, we use the weighting function (8) but allow it to deform according to Kabus' convention.

\subsection{Thirion's Demons}

The first variant of Thirion's Demons algorithm [1] is essentially an approximation to the stationary solution of the following diffusion equation:

$$
\begin{aligned}
\partial_{t} \mathbf{v}(\mathbf{x}, t)-\Delta \mathbf{v}(\mathbf{x}, t) & =\alpha \mathbf{f}(\mathbf{x}, R, F, \mathbf{u}(\mathbf{x}, t)) \\
\mathbf{v}(\mathbf{x}, 0) & =0
\end{aligned}
$$




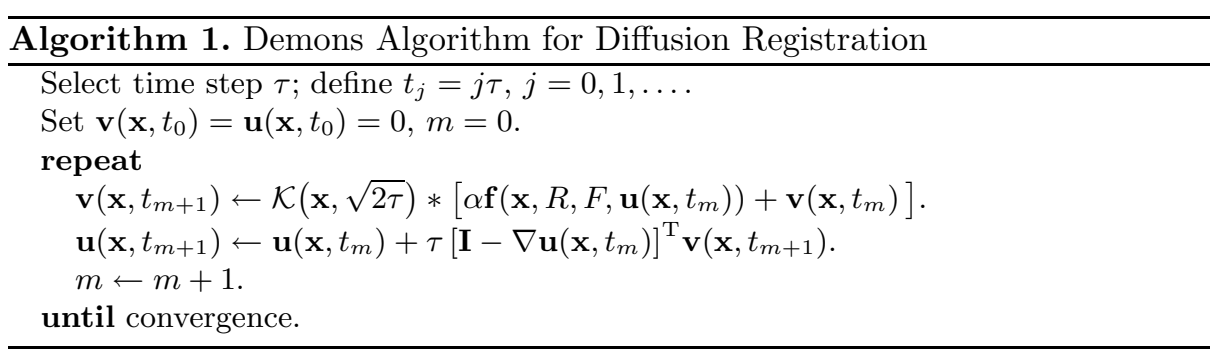

where $\mathbf{x} \in \mathbb{R}^{n}$ and $t \geq 0$. Furthermore, the stationary solution of (9) is equivalent to nonparametric registration with a diffusion regularizer applied to the velocity field. The Demons algorithm exploits the fact that the Gaussian kernel is the Green's function of the diffusion equation, and therefore approximates the stationary solution of (9) by successive Gaussian convolution. Algorithm 1 describes this process, using the notation $\mathcal{K}(\mathbf{x}, \sqrt{2 \tau})$ to indicate a Gaussian kernel at position $\mathbf{x}$ with standard deviation $\sqrt{2 \tau}$, and $*$ to indicate convolution.

Note that in Thirion's description [1] of the Demons algorithm, a normalized version of the sum of squared distances similarity measure is used. Here, we generalize to allow for any similarity measure.

\section{Designing a Locally Adaptive Demons Algorithm}

In order to incorporate locally adaptive regularization into a Demons-style registration framework, we must somehow relate the Euler-Lagrange equations (2) to one or more diffusion equations of the form (9). If this is possible, we can exploit the nature of the Green's function of (9) to construct an approximate solution by successive Gaussian convolution. Unfortunately, due to the nonhomogeneous structure of $\mathcal{A}_{\text {diff }}^{\text {idapt }}$, (which is omitted here for lack of space), there appears to be no direct way to relate the resulting Euler-Lagrange equations to diffusion equations of the form (9).

However, such a relationship can be established if locally adaptive curvature regularizers are constructed. In this section, we show how to construct an imagedriven locally adaptive curvature regularizer, how to relate it to a coupled system of diffusion equations, and finally, how to exploit this relationship to design a locally adaptive Demons algorithm for registration.

\subsection{Locally Adaptive Curvature Regularization}

An image-driven locally adaptive curvature regularizer can be constructed in the same manner as its diffusion counterpart (7) by applying a weighting function to the homogeneous curvature regularizer. This yields:

$$
\mathcal{S}_{\text {curv }}^{\text {id-adapt }}(\mathbf{u})=\frac{1}{2} \int_{\Omega} \beta^{\mathbf{u}}(\mathbf{x})\left(\sum_{j=1}^{n}\left(\Delta u_{j}\right)^{2}\right) d \mathbf{x} .
$$


When the corresponding partial differential operator $\mathcal{A}_{\text {curv }}^{\text {id-apt }}$ is determined and applied to the velocity field, the corresponding Euler-Lagrange equation can be written as:

$$
\Delta\left[\beta^{\mathbf{u}}(\mathbf{x}) \Delta \mathbf{v}\right]=\frac{1}{2}\left(\sum_{j=1}^{n}\left(\Delta v_{j}\right)^{2}\right) \nabla \beta^{\mathbf{u}}(\mathbf{x})+\alpha \mathbf{f}(\mathbf{x}, R, F, \mathbf{u}(\mathbf{x})) .
$$

\subsection{Coupled PDE System}

The structure of the left hand side of the Euler-Lagrange equation (11) allows us to relate the solution of (11) to the stationary solution of a coupled system of diffusion equations. This coupled system is given by:

$$
\begin{aligned}
& \partial_{t} \mathbf{w}(\mathbf{x}, t)-\Delta \mathbf{w}(\mathbf{x}, t)=\sqrt{\alpha} \mathbf{f}(\mathbf{x}, R, F, \mathbf{u}(\mathbf{x}, t))-\frac{\sqrt{\alpha}}{2}\left(\sum_{j=1}^{n}\left(w_{j}(\mathbf{x}, t)\right)^{2}\right) \nabla\left[1 / \beta^{\mathbf{u}(\mathbf{x}, t)}(\mathbf{x})\right], \\
& \partial_{t} \mathbf{v}(\mathbf{x}, t)-\Delta \mathbf{v}(\mathbf{x}, t)=\sqrt{\alpha} \mathbf{w}(\mathbf{x}, t) / \beta^{\mathbf{u}(\mathbf{x}, t)}(\mathbf{x}), \\
& \mathbf{w}(\mathbf{x}, 0)=\mathbf{v}(\mathbf{x}, 0)=0 .
\end{aligned}
$$

In order to establish the relationship between the stationary solution of (12)-(13) and the solution of (11), we first note that the stationary solution of (12)-(13) satisfies $\partial_{t} \mathbf{w}=\partial_{t} \mathbf{v}=0$. Therefore, as $t \rightarrow \infty$, we see that solving for $\mathbf{w}(\mathbf{x}, t)$ in (13) and substituting the result into (12) yields (11).

\subsection{Locally Adaptive Demons}

We have shown for the locally adaptive curvature regularizer that the solution to the Euler-Lagrange equation is equivalent to the stationary solution of a coupled system of diffusion equations. Therefore, we can exploit the structure of the Green's function of the diffusion equation in order to define a Demons-style algorithm for registration with this adaptive regularizer. Algorithm 2 lists the resulting Demons algorithm.

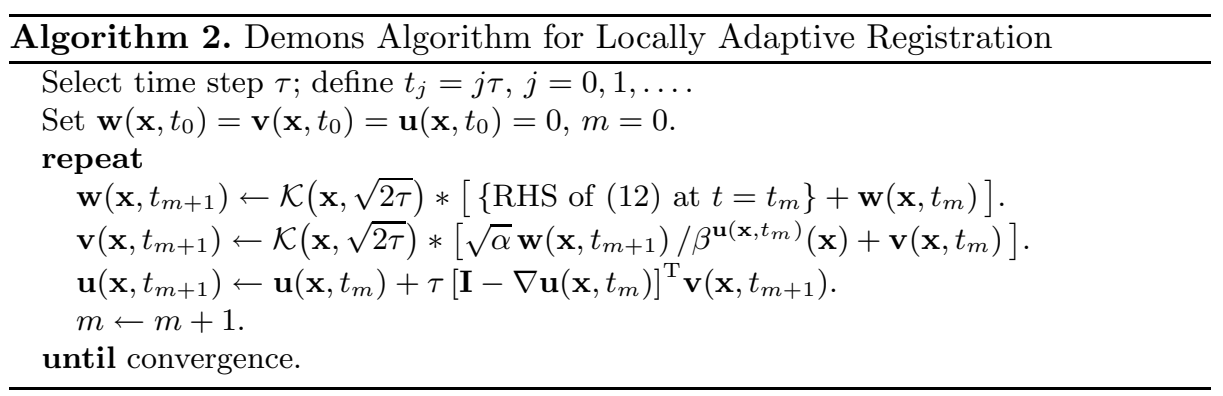




\section{Pedagogical Example}

To illustrate the behavior of the locally adaptive Demons algorithm, we consider a pedagogical example of registering two ellipses. Figure 1 shows two $100 \times 100$ images of an ellipse with smoothed edges; the floating ellipse has left edge that is five pixels to the left of the left edge of the reference ellipse. The right edges of both ellipses are in the same location.

In a simple experiment, we registered the ellipses using the standard and locally adaptive Demons algorithms. Figures 1(d) and 1(c) show that each algorithm warps the edge of the floating ellipse in a similar manner, but that interior of the floating ellipse is warped at different rates. Figure 1(e) affirms this point: the use of locally adaptive Demons with $\epsilon=10^{-2} / \epsilon=1$ causes the interior of the ellipse to be less/more affected by the ellipse edge than the standard Demons algorithm.

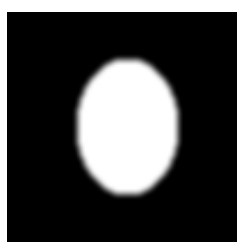

(a) Floating

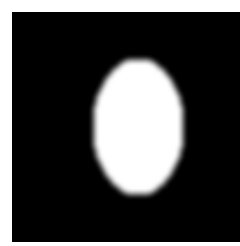

(b) Reference

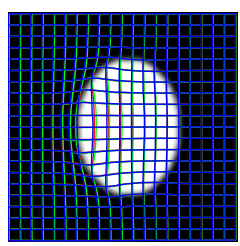

(c) Warp Fields

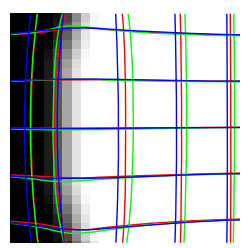

(d) Zoomed View

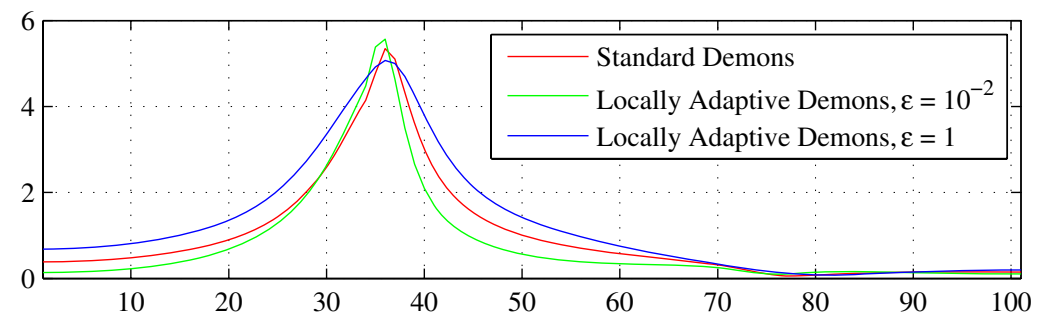

(e) Horizontal displacement component across the middle row of the floating image. $X$ axis of plot corresponds to pixel location.

Fig. 1. Pedagogical example: warp fields correspond to standard Demons algorithm with $\alpha=10^{-4}$ (red), locally adaptive Demons algorithm with $\alpha=10^{-4}$ and $\epsilon=10^{-2}$ (green), and locally adaptive Demons algorithm with $\alpha=10^{-3}$ and $\epsilon=1$ (blue)

\section{Serial Chest CT Experiment}

A cursory analysis of Fig. 1(e) suggests that the locally adaptive Demons algorithm can be tuned to enable a range of behavior in how deformations are smoothed near edges in the image. To explore this behavior on a practical example, we register serial chest $\mathrm{CT}$ examinations of 18 patients with lung nodules. 
Table 1. Statistics of TRE across all nodules, ATRE across all cases, and ECS for rigid registration and nonrigid registration via standard and locally adaptive Demons

\begin{tabular}{|l|c|c|c|}
\hline Statistic & Rigid only & $\begin{array}{c}\text { Rigid }+ \\
\text { standard Demons }\end{array}$ & $\begin{array}{c}\text { Rigid + locally } \\
\text { adaptive Demons }\end{array}$ \\
\hline \hline Mean TRE $(\mathrm{mm})$ & 8.5 & 2.6 & 2.5 \\
Median TRE (mm) & 7.2 & 2.0 & 1.8 \\
Std. dev. TRE (mm) & 6.5 & 2.2 & 2.1 \\
\hline Mean ATRE (mm) & 8.4 & 2.3 & 2.1 \\
Median ATRE (mm) & 7.8 & 2.4 & 1.9 \\
Std. dev. ATRE (mm) & 4.5 & 1.6 & 1.5 \\
\hline ECS Percentiles (25\%,50\%,75\%) & & $(53.0,56.4,56.5)$ & $(17.2,19.0,21.8)$ \\
\hline
\end{tabular}

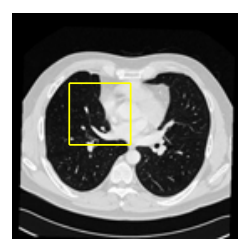

(a)

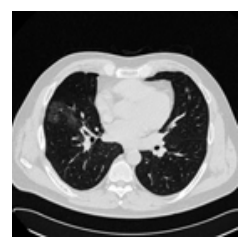

(b)

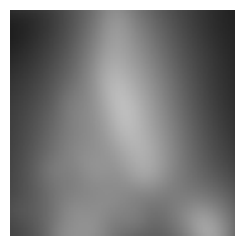

(c)

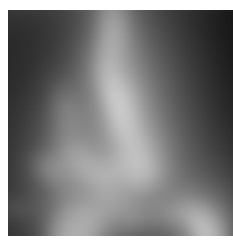

(d)

Fig. 2. Chest CT registration: (a) Axial slice from prior volume; (b) Axial slice from current volume; (c) $\log (1+\|\nabla \mathbf{u}\|)$ from yellow subregion of (a) using standard Demons; (d) $\log (1+\|\nabla \mathbf{u}\|)$ from yellow subregion of (a) using locally adaptive Demons

To establish a set of ground truth points that can be used to measure target registration error (TRE), we manually identified the centers of lung nodules less than $6 \mathrm{~mm}$ in diameter that are observable in both the prior and current images of a patient. This yielded from 4-20 ground truth points for each patient. We resampled the images to approximately $3 \times 3 \times 3 \mathrm{~mm}^{3}$ isotropic resolution and performed a rigid preregistration step. We then performed nonrigid registration using the standard and locally adaptive Demons algorithms with various values of $\alpha$ and $\epsilon$. Registration was performed in a multiresolution pyramid at four resolution levels.

Computational requirements of each algorithm were measured in terms of effective convolution steps (ECS). A unit of ECS is defined as the amount of computation required to convolve a vector field at the finest resolution level with a Gaussian kernel. Hence, 50 iterations of standard Demons at each of the two finest levels requires $50+(1 / 8) * 50=56.25$ ECS. Adaptive Demons would require twice as many Gaussian convolution steps, yielding 112.5 ECS.

Table 1 shows statistics on the TRE values across all nodules, aggregate TRE (ATRE) values across all cases for the best performing algorithms (standard: $\alpha=$ $10^{-2}$; locally adaptive: $\alpha=10^{-2}, \epsilon=10^{-2}$ ), and ECS. ATRE for a particular patient case is defined as the median TRE value for all nodules in the case. Figure 2 illustrates an axial slice from the prior and current CT images of one 
patient, along with images of $\log (1+\|\nabla \mathbf{u}\|)$ for the deformations resulting from the standard and locally adaptive Demons algorithms.

\section{Conclusion}

We have shown how the Demons algorithm can be generalized to handle a locally adaptive regularizer. This is done by constructing an image-driven locally adaptive version of the curvature regularizer, and then relating the solution of the Euler-Lagrange equations for registration to the stationary solution of a pair of diffusion equations. The proposed algorithm is easy to implement and exhibits linear complexity in the number of pixels/voxels. Experiments on serial registration of chest CT images of patients with lung nodules show an improvement in TRE as well as a reduction in the amount of computational effort required.

\section{References}

1. Thirion, J.P.: Image matching as a diffusion process: an analogy with maxwell's demons. Medical Image Analysis 2(3), 243-260 (1998)

2. Modersitzki, J.: Numerical Methods for Image Registration. Oxford University Press, Oxford (2004)

3. Alvarez, L., Esclarín, J., Lefébure, M., Sánchez, J.: A PDE model for computing the optical flow. In: Proceedings XVI Congreso de Ecuaciones Diferenciales y Aplicaciones, September 1999, pp. 1349-1356 (1999)

4. Weickert, J., Bruhn, A., Brox, T., Papenberg, N.: A survey on variational optic flow methods for small displacements. In: Mathematical Models for Registration and Applications to Medical Imaging, pp. 103-136. Springer, Heidelberg (2006)

5. Bro-Nielsen, M.: Medical Image Registration and Surgery Simulation. PhD thesis, Technical University of Denmark, DTU (1996)

6. Crum, W.R., Tanner, C., Hawkes, D.J.: Anisotropic multi-scale fluid registration: Evaluation in magnetic resonance breast imaging. Physics in Medicine and Biology 50(21), 5153-5174 (2005)

7. Cahill, N.D., Noble, J.A., Hawkes, D.J.: Fourier methods for nonparametric image registration. In: Proc. CVPR Workshop on Image Registration and Fusion, June 2007, pp. 1-8 (2007)

8. Cahill, N.D., Noble, J.A., Hawkes, D.J.: Demons algorithms for fluid and curvature registration. In: Proc. International Symposium on Biomedical Imaging (June 2009)

9. Charbonnier, P., Blanc-Féraud, L., Aubert, G., Barlaud, M.: Two determinstic half-quadratic regularization algorithms for computed imaging. In: Proc. 1994 IEEE International Conference on Image Processing., vol. 2, pp. 168-172 (1994)

10. Bruhn, A., Weickert, J., Kohlberger, T., Schnörr, C.: A multigrid platform for real-time motion computation with discontinuity-preserving variational methods. International Journal of Computer Vision 70(3), 257-277 (2006)

11. Kabus, S.: Multiple-Material Variational Image Registration. PhD thesis, der Universität zu Lübeck (October 2006) 